prescription of an occupational disease can be based upon isolated clinical observations only. We live now in an era of planned observation of populations. It is difficult enough to establish a non-specific disease as an occupational disease in an industry by carefully planned statistical record, without wasting time and effort on the collection of clinical cases devoid of the necessary data from which valid conclusions can be drawn. It is not necessary to designate such work as mathematical.

Baader rightly deplores the failure of physicians and surgeons to inquire into the occupation of their patients and instances cases where mis-diagnoses led to a continuance of hazardous work.

He rightly condemns the unrewarding diagnusis of gas-poisoning and the ambiguous designations of occupations, which do not reveal the nature of the patient's occupational exposure.

He approves the statement of Flury that poisons only "stimulate or inhibit the function of single organs and do not produce functions which are normally not characteristic of them" and illustrates from the chronic nephritis of lead, syphilis, vascular disease, or the endstage of acute nephritis; from bronchitis and bronchopneumonia brought about by irritant gases or infection; from bladder tumours of aniline workers (sic) and bronchogenic cancer among miners of pitch-blend; from disturbances of heat regulation by $\mathrm{CO}, \mathrm{CS}_{2}, \mathrm{CCl}_{4}$, and $\mathrm{C}_{6} \mathrm{H}_{6}$ which might be attributed wrongly to infection.

It must be admitted that the doctrine is at present almost unassailable but must ultimately be annihilated as more and more knowledge is gathered of the intracellular mechanisms of toxic action. The crude methods of today must soon be replaced by ways of distinguishing between, for example, the cellular disturbances brought about by different carcinogenic agents even though the ultimate tumours induced are clinically and histologically indistinguishable. It is true that there are more ways than one of killing a cat but the ways are distinguishable and more or less avoidable. The differences in reversibility of the effects of toxic agents should give us pause in equating the intermediate stages even when the final stages may appear identical. The long latent periods of many occupational diseases, the apparently contradictory results of the same toxic agent, the loss of resistances to many chemical noxa after more or less lengthy exposure, all await explanation which Flury's doctrine does not help us to attain. But he was writing over 30 years ago.

Baader gives the 1952 lists and the projected lists of prescribed diseases enacted in the German Federal Republic; the 1957 list in the East German Republic; lists in the Saar (1954), Austria (1955), and Switzerland $(1952,1956)$. The German lists are generally similar to those in the United Kingdom. The prescription of tearfractures of spinal processes and of asbestosis combined with lung carcinoma rings strangely in British ears.

A difference from the more specific prescription used in this country for tumours of the bladder is the German formula of "Cancer or other new growths as well as changes in the mucous membrane of the urinary tract brought about by aromatic amines".

Another aspect of some interest in German prescrip- tions is the inclusion of the phrase "... which necessitate a change of occupation or complete cessation of all work". It may not be generally known that prescription in Switzerland includes a list of organic and inorganic recognized causes of "dangerous illnesses", and block designations such as "Chlorinated ethers of the aliphatic series" or "Halogenated aliphatic hydrocarbons".

The treatment by Baader of the extensive field from the clinical point of view is, as would be expected, comprehensive and in the classical manner.

The book is not easy to read consecutively but of its value as an up-to-date reference volume there can be no doubt.

The literature quoted is extensive even if, except in one or two fields, Britain does not figure very noticeably.

Professor Baader is to be congratulated on a notable achievement.

\section{W. Goldblatt}

Occupational Disease in California Attributed to Pesticides and Agricultural Chemicals, 1959. (Pp. 30.) California: State Department of Public Health. 1961.

Under Californian law a doctor who attends a patient injured at work and whose disability extends beyond the day of the injury must file a report. A total of 1,093 such "first reports" on cases of illness attributed to pesticides during 1959 are analysed and discussed in this publication.

About $42 \%$ of the cases were poisoning by organophosphorus insecticides. The only fatalities during the year were two men engaged in fumigation, one with carbon tetrachloride and the other with methyl bromide. Although "first reports" may not always give the final diagnosis these data from one of the most intensively developed and regulated agricultural areas of the world are instructive.

Complimentary copies are available upon request, within limitations of the supply, from the Bureau of Health Education, California State Department of Public Health, 2151 Berkeley Way, Berkeley 4, California.

\section{J. M. BARNeS}

Micro-organisms as Allies: The Industrial Use of Fungi and Bacteria. By C. L. Duddington. (Pp. 256; 21 figures. 25s.) London: Faber and Faber. 1961.

This book deals with the many and varied ways in which bacteria and moulds are used in industry. The opening chapter entitled "The Livestock of Industry" describes the work of Louis Pasteur, generally accepted as the pioneer in this field, and details the research he carried out for the French wine and silk trade and how vast industries have been founded on the efforts of Pasteur and subsequent microbiologists.

As might be expected, a considerable amount of space has been given to the brewing process, and the yeast used in this trade is cultured with great care in very elaborate laboratories. The main differences between top fermentation, where the yeast grows on the surface of the wort to produce most types of British beers, and bottom fermentation, where the yeast lives at the bottom 
of the vat under anaerobic conditions, yielding lager beer, are explained.

It is interesting to read that the wine trade has such antiquity dating from Mesolithic time, more than ten thousand years ago, and to follow its progress to modern commercial production where countries and districts specialize to market a characteristic product.

The diseases which affect the vine are many and varied and, until the advent of the newer insecticides and fungicides, the struggle has been intense, with the vine grower barely holding his own in this war of attrition. The commercial fermentation of the juice of the grape to yield wine is performed by the wine yeast Saccharomyces ellipsoideus and the growth of the wild yeast and bacteria is discouraged by the addition of sulphur dioxide to the "must" (grape juice) before fermentation.

The section of the book which deals with the famous vintage wines is fascinating and differences in vines, soils, and climates, which combine to give the characteristic types of great wines, are stressed.

It is recorded that Arrach, a spirit made by distilling the juice of the coconut palm, was known in India at least 800 years before the Christian era.

The Irish are given the credit for being the first race to distil whiskey from fermented grain and this preceded the production of brandy by the French. The legend has it that the Irish were originally taught whiskey-making by St. Patrick.

The use of yeast by the baker to lighten his bread has considerable antiquity, as the process was developed by the ancient Egyptians and the same method is used commercially today in all countries of the world.

The chapter on moulds and antibiotics chronicles the tremendous developments in this field in recent years, since the original observation of Fleming when he described the inhibition of cultures of Staphylococcus aureus by Penicillium. However, it is pointed out that the Chinese used soy flour poultices to treat infected wounds and the Ebers Papyrus of 1500 B.C. extolled the virtues of "yeast of sweet beer" for similar use.

This book is of importance to doctors who work in industry for great care has been taken to give a concise and accurate description of all the processes in which micro-organisms play a part in industry.

No mention has been made of any occupational diseases attributable to these allies of industry but no doubt the author would consider such information as outside his terms of reference.

John F. Eustace

Toxicology and Biochemistry of Aromatic Hydrocarbons. By H. W. Gerarde. (Pp. xiii $+329 ; 89$ figures + 63 tables. 30s.) Amsterdam: Elsevier. 1960.

This book is divided into two parts. The first, General Considerations, includes a brief account of the nomenclature and structure of the aromatic hydrocarbons, also the relationship between chemical constitution and physical properties, taste, smell, toxicity, and biochemistry. The biochemistry of these compounds includes sections on absorption, tissue distribution, excretion, and metabolism. The final chapter of the section discusses what constitutes an industrial hazard, threshold limits, and treatment.

The second part deals in more detail with 20 aromatic hydrocarbons commonly encountered industrially, under the headings "physical and chemical properties", "sources and probable modes of contact", "analytical methods", "toxicity", "biochemistry", "prevention, detection and treatment of exposure". A brief section on the carcinogenicity of the polycyclic hydrocarbons is included, and an appendix containing a glossary of terms commonly encountered in the petroleum industry.

The industrial hygienist and toxicologist, for whom it is primarily intended, should find this a valuable handbook, containing in one volume much of the chemical, biochemical, and toxicological data he requires, set out in tabular form and including previously unpublished work of the author.

The book is printed on good quality paper, but the binding of the review copy was inadequate, loose pages appearing after minimal usage.

\section{J. Threlfall}

Air Pollution. (Monograph Series No. 46.) (Pp. 442; 4 figures. 40s.) Geneva: World Health Organization. 1961.

In the historical review by E. C. Halliday which opens this Monograph there is a clear indication of the rapidly mounting number of papers on air pollution which have been published since the end of the Second World War. There have as yet been few books summarizing these developments, but this well-produced volume goes a considerable way towards bridging the gap. It is intended mainly for the benefit of public health officials and it brings together a series of 14 articles by authors from seven different countries. It suffers from the inevitable time lag involved in the preparation of books with multiple authorship, but it provides a useful survey of world trends in research up to 1957 . The coverage is wide, but for British readers it is liable to set a somewhat false perspective. Care has been taken to exclude purely occupational hazards, but throughout there is a tendency to emphasize the importance of industrial emissions. Domestic sources of pollution receive very little attention and nowhere is there any detailed account of the distribution of smoke and sulphur dioxide within large towns.

The individual sections vary widely in length and quality. Some contain a profusion of detail worthy of a reference work whilst others are written in terms too general even for a broad survey. One of the longest and most valuable is by Morris Katz, concerning the physical and chemical nature of air pollution. This is an admirable discussion of the several types of pollution which may be found in urban atmospheres with particular reference to conditions in Great Britain, Los Angeles, and the Detroit-Windsor area. It is followed by an equally long discussion by Harry Heimann on health aspects in which considerable emphasis is placed on the major episodes of high pollution. The amount of overlap which occurs between sections is quite small, but a table showing the average composition of the atmosphere appears in each of these two contributions and the Los 